\title{
COSMIC BACKGROUND RADIATION
}

To reduce the size of this section's PostScript file, we have divided it into two PostScript files. We present the following index:

PART 1

\begin{tabular}{cll}
\hline Page $\#$ & \multicolumn{2}{l}{ Section name } \\
\hline 1 & 19.1 & Introduction \\
1 & 19.2 & The CMB frequency spectrum \\
\hline \hline
\end{tabular}

PART 2

\begin{tabular}{|c|c|}
\hline Page \# & Section name \\
\hline 6 & 19.3 Deviations from isotropy \\
\hline 11 & References \\
\hline
\end{tabular}


Revised April 1998 by G.F. Smoot (LBNL) and D. Scott (University of British Columbia).

\subsection{Introduction}

The observed cosmic microwave background (CMB) radiation provides strong evidence for the hot big bang. The success of primordial nucleosynthesis calculations (see Sec. 16, "Big-bang nucleosynthesis") requires a cosmic background radiation (CBR) characterized by a temperature $k T \sim 1 \mathrm{MeV}$ at a redshift of $z \simeq 10^{9}$. In their pioneering work, Gamow, Alpher, and Herman [1] realized this and predicted the existence of a faint residual relic, primordial radiation, with a present temperature of a few degrees. The observed CMB is interpreted as the current manifestation of the required CBR.

The CMB was serendipitously discovered by Penzias and Wilson [2] in 1965. Its spectrum is well characterized by a $2.73 \pm 0.01 \mathrm{~K}$ black-body (Planckian) spectrum over more than three decades in frequency (see Fig. 19.1). A non-interacting Planckian distribution of temperature $T_{i}$ at redshift $z_{i}$ transforms with the universal expansion to another Planckian distribution at redshift $z_{r}$ with temperature $T_{r} /\left(1+z_{r}\right)=T_{i} /\left(1+z_{i}\right)$. Hence thermal equilibrium, once established (e.g. at the nucleosynthesis epoch), is preserved by the expansion, in spite of the fact that photons decoupled from matter at early times. Because there are about $10^{9}$ photons per nucleon, the transition from the ionized primordial plasma to neutral atoms at $z \sim 1000$ does not significantly alter the CBR spectrum [3].

\subsection{The CMB frequency spectrum}

The remarkable precision with which the CMB spectrum is fitted by a Planckian distribution provides limits on possible energy releases in the early Universe, at roughly the fractional level of $10^{-4}$ of the CBR energy, for redshifts $\lesssim 10^{7}$ (corresponding to epochs $\gtrsim 1$ year). The following three important classes of theoretical spectral distortions (see Fig. 19.2) generally correspond to energy releases at different epochs. The distortion results from the CBR photon interactions with a hot electron gas at temperature $T_{e}$.

19.2.1. Compton distortion: Late energy release $\left(z \lesssim 10^{5}\right)$. Compton scattering $\left(\gamma e \rightarrow \gamma^{\prime} e^{\prime}\right)$ of the CBR photons by a hot electron gas creates spectral distortions by transfering energy from the electrons to the photons. Compton scattering cannot achieve thermal equilibrium for $y<1$, where

$$
y=\int_{0}^{z} \frac{k T_{e}\left(z^{\prime}\right)-k T_{\gamma}\left(z^{\prime}\right)}{m_{e} c^{2}} \sigma_{T} n_{e}\left(z^{\prime}\right) c \frac{d t}{d z^{\prime}} d z^{\prime}
$$

is the integral of the number of interactions, $\sigma_{T} n_{e}(z) c d t$, times the mean-fractional photon-energy change per collision [4]. For $T_{e} \gg T_{\gamma} y$ is also proportional to the integral of the electron pressure $n_{e} k T_{e}$ along the line of sight. For standard thermal histories $y<1$ for epochs later than $z \simeq 10^{5}$.

CITATIOn: C. Caso et al., European Physical Journal C3, 1 (1998) 


\section{19. Cosmic background radiation}

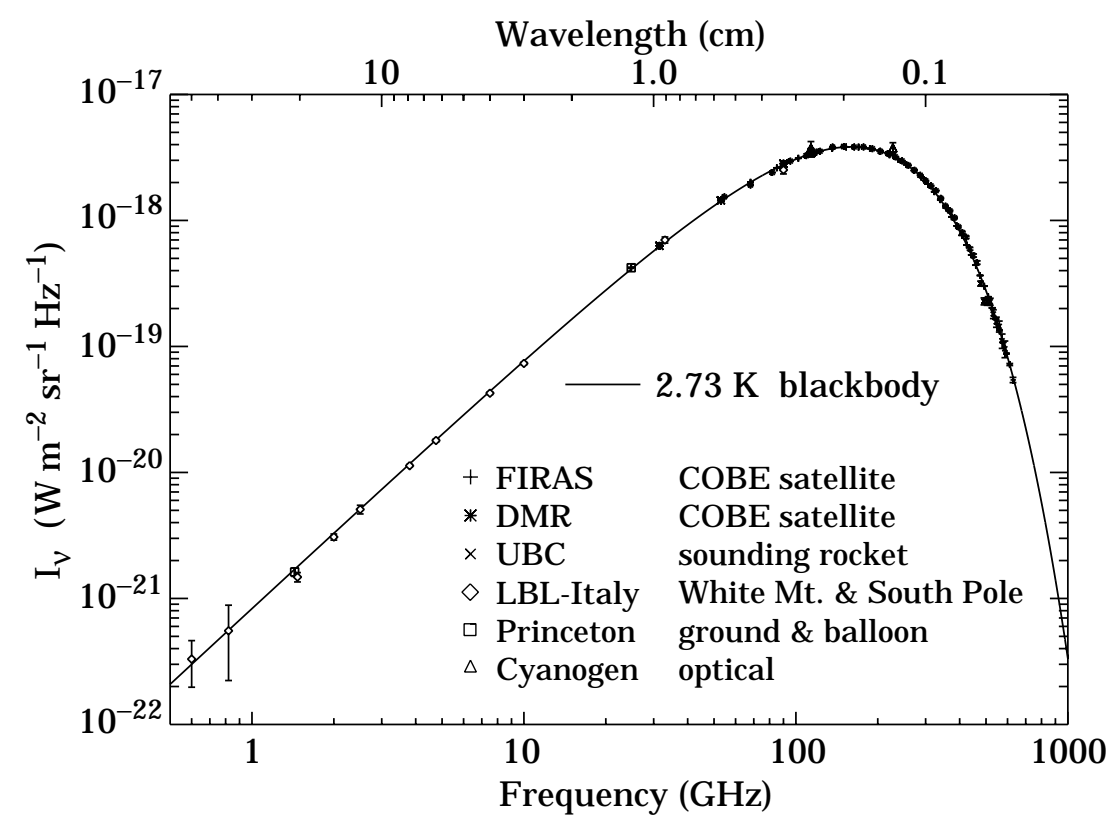

Figure 19.1: Precise measurements of the CMB spectrum. The line represents a $2.73 \mathrm{~K}$ blackbody, which describes the spectrum very well, especially around the peak of intensity. The spectrum is less well constrained at $10 \mathrm{~cm}$ and longer wavelengths. (References for this figure are at the end of this section under "CMB Spectrum References.")

The resulting CMB distortion is a temperature decrement

$$
\Delta T_{\mathrm{RJ}}=-2 y T_{\gamma}
$$

in the Rayleigh-Jeans $(h \nu / k T \ll 1)$ portion of the spectrum, and a rapid rise in temperature in the Wien $(h \nu / k T \gg 1)$ region, i.e. photons are shifted from low to high frequencies. The magnitude of the distortion is related to the total energy transfer [4] $\Delta E$ by

$$
\Delta E / E_{\mathrm{CBR}}=e^{4 y}-1 \simeq 4 y .
$$

A prime candidate for producing a Comptonized spectrum is a hot intergalactic medium. A hot $\left(T_{e}>10^{5} \mathrm{~K}\right)$ medium in clusters of galaxies can and does produce a partially Comptonized spectrum as seen through the cluster, known as the Sunyaev-Zel'dovich effect. Based upon X-ray data, the predicted large angular scale total combined effect of the hot intracluster medium should produce $y \lesssim 10^{-6}[5]$. 


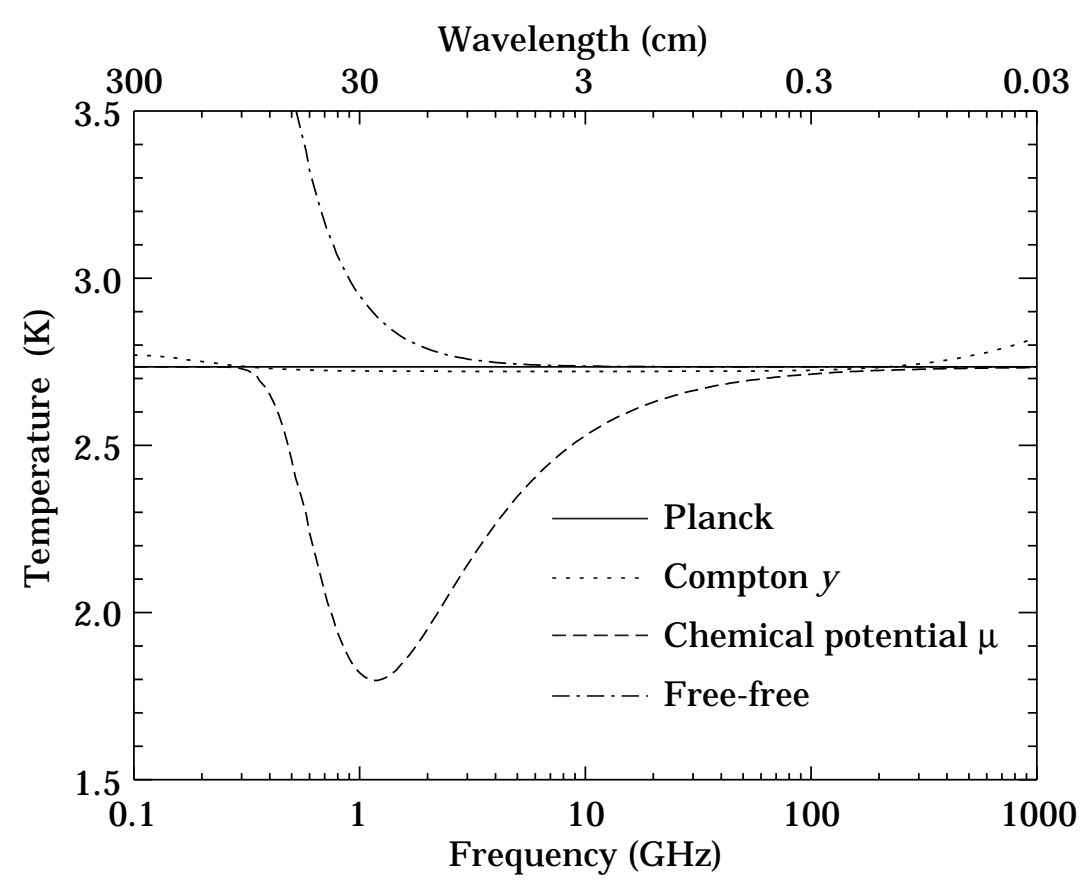

Figure 19.2: The shapes of expected, but so far unobserved, CMB distortions, resulting from energy-releasing processes at different epochs.

19.2.2. Bose-Einstein or chemical potential distortion: Early energy release $\left(z \sim 10^{5}-10^{7}\right)$. After many Compton scatterings $(y>1)$, the photons and electrons will reach statistical (not thermodynamic) equilibrium, because Compton scattering conserves photon number. This equilibrium is described by the Bose-Einstein distribution with non-zero chemical potential:

$$
n=\frac{1}{e^{x+\mu_{0}}-1},
$$

where $x \equiv h \nu / k T$ and $\mu_{0} \simeq 1.4 \Delta E / E_{\mathrm{CBR}}$, with $\mu_{0}$ being the dimensionless chemical potential that is required.

The collisions of electrons with nuclei in the plasma produce free-free (thermal bremsstrahlung) radiation: $e Z \rightarrow e Z \gamma$. Free-free emission thermalizes the spectrum to the plasma temperature at long wavelengths. Including this effect, the chemical potential becomes frequency-dependent,

$$
\mu(x)=\mu_{0} e^{-2 x_{b} / x},
$$

where $x_{b}$ is the transition frequency at which Compton scattering of photons to higher frequencies is balanced by free-free creation of new photons. The resulting spectrum has a sharp drop in brightness temperature at centimeter wavelengths [6]. The minimum wavelength is determined by $\Omega_{B}$. 


\section{19. Cosmic background radiation}

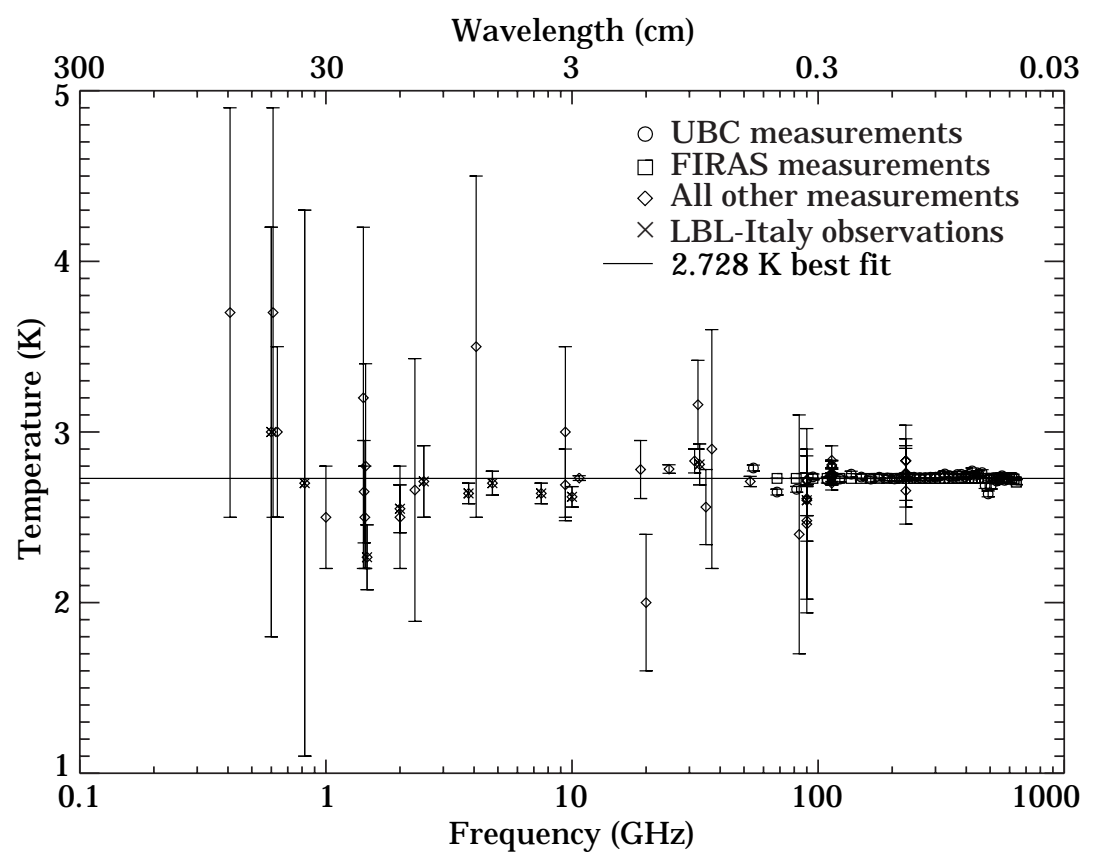

Figure 19.3: Observed thermodynamic temperature as a function frequency.

The equilibrium Bose-Einstein distribution results from the oldest non-equilibrium processes $\left(10^{5}<z<10^{7}\right)$, such as the decay of relic particles or primordial inhomogeneities. Note that free-free emission (thermal bremsstrahlung) and radiativeCompton scattering effectively erase any distortions [7] to a Planckian spectrum for epochs earlier than $z \sim 10^{7}$.

19.2.3. Free-free distortion: Very late energy release $\left(z \ll 10^{3}\right)$. Free-free emission can create rather than erase spectral distortion in the late Universe, for recent reionization $\left(z<10^{3}\right)$ and from a warm intergalactic medium. The distortion arises because of the lack of Comptonization at recent epochs. The effect on the present-day CMB spectrum is described by

$$
\Delta T_{f f}=T_{\gamma} Y_{f f} / x^{2},
$$

where $T_{\gamma}$ is the undistorted photon temperature, $x$ is the dimensionless frequency, and $Y_{f f} / x^{2}$ is the optical depth to free-free emission:

$$
Y_{f f}=\int_{0}^{z} \frac{T_{e}\left(z^{\prime}\right)-T_{\gamma}\left(z^{\prime}\right)}{T_{e}\left(z^{\prime}\right)} \frac{8 \pi e^{6} h^{2} n_{e}^{2} g}{3 m_{e}\left(k T_{\gamma}\right)^{3} \sqrt{6 \pi m_{e} k T_{e}}} \frac{d t}{d z^{\prime}} d z^{\prime} .
$$

Here $h$ is Planck's constant, $n_{e}$ is the electron density and $g$ is the Gaunt factor [8]. 


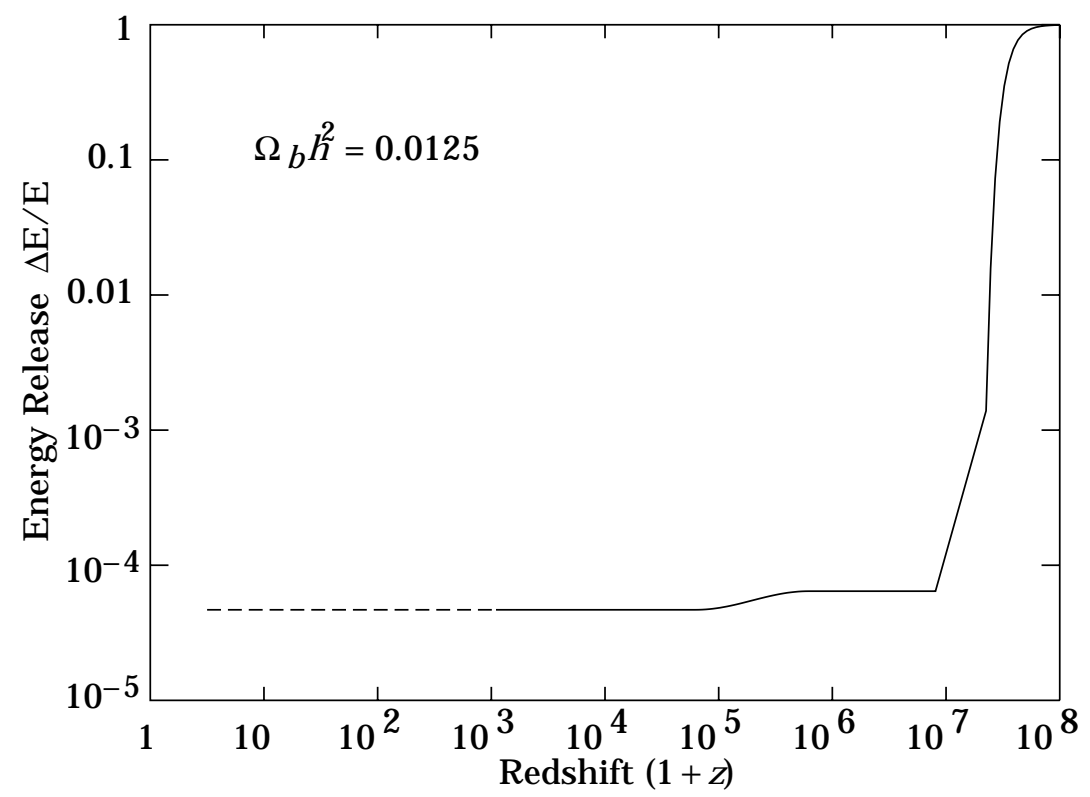

Figure 19.4: Upper Limits $(95 \% \mathrm{CL})$ on fractional energy $\left(\Delta E / E_{\mathrm{CBR}}\right)$ releases from processes at different epochs as set by resulting lack of CMB spectral distortions. These can be translated into constraints on the mass, lifetime and photon branching ratio of unstable relic particles, with some additional dependence on cosmological parameters such as $\Omega_{B}[9,10]$.

19.2.4. Spectrum summary: The CMB spectrum is consistent with a blackbody spectrum over more than three decades of frequency around the peak. A least-squares fit to all CMB measurements yields:

$$
\begin{array}{ll}
T_{\gamma}=2.728 \pm 0.002 \mathrm{~K} & (1 \sigma \text { error }) \\
n_{\gamma}=\left(2 \zeta(3) / \pi^{2}\right) T_{\gamma}^{3} \simeq 412 \mathrm{~cm}^{-3} \\
\rho_{\gamma}=\left(\pi^{2} / 15\right) T_{\gamma}^{4} \simeq 4.68 \times 10^{-34} \mathrm{~g} \mathrm{~cm}^{-3} \simeq 0.262 \mathrm{eV} \mathrm{cm}^{-3} \\
|y|<1.2 \times 10^{-5} \quad(95 \% \mathrm{CL}) \\
\left|\mu_{0}\right|<9 \times 10^{-5} \quad(95 \% \mathrm{CL}) \\
\left|Y_{f f}\right|<1.9 \times 10^{-5} \quad(95 \% \mathrm{CL})
\end{array}
$$

The limits here [11] correspond to limits [11-13] on energetic processes $\Delta E / E_{\mathrm{CBR}}<$ $2 \times 10^{-4}$ occurring between redshifts $10^{3}$ and $5 \times 10^{6}$ (see Fig. 19.4). The best-fit temperature from the COBE FIRAS experiment is $T_{\gamma}=2.728 \pm 0.002 \mathrm{~K}$ [11]. 